Periodica Mothemation Hungarioa Vol. 3 (3-4), (1973), pp. 221-225.

\title{
ON THE EXISTENCE OF TRIANGULATED SPHERES IN 3-GRAPHS, AND RELATED PROBLEMS
}

\author{
by \\ V. T. SÓs, P. ERDÓs (Budapest) and W. G. BROWN (Montreal)
}

To the memory of A. RENYI

1. An r-graph, $H^{(r)}$ consists of a set $V\left(H^{(r)}\right)$ of vertices, and a elass $E\left(H^{(r)}\right.$ of $r$-subsets of $V\left(H^{(r)}\right)$ (i.e. unordered $r$-tuples of vertices). We shall use various letters (in place of $H$ ) to name $r$-gxaphs, but the superscript $(r)$ will always be included; except possibly when $r=2-$ a 2-graph is simply a graph. If we follow the symbol $H^{(r)}$ by $(n)$ or $(n ; k)$ it will mean that the $r$-graph has exactly $n$ vertices, and, in the second case, at least $k r$-tuples (as we shall name the members of $\left.E\left(H^{(r)}\right)\right)$. The letter $G$ is reserved to denote all $r$-graphs with the properties indicated by any appendices to the symbol: thus $G^{(r)}$ represents any $r$-graph; $G^{(r)}(n)$ any $G^{(r)}$ with $n$ vertices; and $G^{(r)}(n ; k)$ any $G^{(r)}(n)$ with at least $k$-tuples.

The extremal theory of graphs was initiated by P. Turis $[20,21]$ : he determined for every positive integer $t$ the smallest integer $k$ for which every $G(n ; k)$ contains a complete $t$-gon $\left(\right.$ i.e. a $G\left(t ;\left(\begin{array}{l}t \\ 2\end{array}\right)\right)$. Extremal problems for ordinary graphs have been studied extensively [2-9, eto.]; many general results have been obtained, but many unsolved problems remain. For $r$-graphs very little is known $[5,11]$. Even the original problem of TURAin [20] (to determine the smallest $k$ for which for fixed $t$, every $G^{(r)}(n ; k)$ contains all $\left(\begin{array}{l}t \\ r\end{array}\right)$ $r$-tuples which can be formed from some $t$ vertices) remains unsolved $[14,16]$.

In this paper we shall confine our interest to 3 -graphs. The problem described in the title represents an analogue of the well known property of graphs that any $G(n ; n)$ contains a polygon. That result could be restated, in topological terms, as saying that any simplicial 1-complex with at least as many 1-simplexes as 0 -simplexes must contain a triangulation of the 1-sphere. (It is best possible in the sense that there exist $G(n ; n-1)$ 's containing no polygon.) In Theorem 3 we shall determine asymptotically the maximum number of 2-simplexes a simplicial 2-complex may contain without containing 
a subcomplex which is a triangulation of the 2-sphere. More precisely, we shall prove that there exist constants $c_{1}$ and $c_{2}$ such that every $G^{(3)}\left(n ; c_{2} n^{3 / 2}\right)$ contains a double pyramid (i.e. a polytope whose faces are all triangles having two of their vertices as adjacent vertices of a fixed polygon, and whose third vertex is one of two fixed points); but that some $G^{(3)}\left(n ; r_{1} n^{3 z}\right)$ contains no triangulation of the sphere. Also, we discuss several related results.

2. Extremal numbers. Let $H$ be any fixed family of $r$-graphs. Then $\operatorname{ex}(n ; H)$ will denote the largest integer $k$ for which there exists a $G^{(n)}(n ; k)$ containing none of the members of $H$ as a sub-r-graph. (When $H$ eonsists of a single $r$-graph $H^{(n)}$ we may abbreviate $\operatorname{ex}\left(n ;\left\{H^{(r)}\right\}\right)$ to $\left.\operatorname{ex}\left(n ; H^{(r)}\right)\right)$. For s lens than $r$ the s-tuples of an $r$-graph will be any set of $s$ vertices; in particular, the edges will be any unordered pair of vertices. The star of an $s$-tuple $S$ in a $G^{(\prime)}$ $(s<r)$ will be the $(r-s)$-graph whose vertices are all vertices of the $G^{(r)}$ not in $S$, and whose $(r-s)$-tuples are all sets $U-S$ where $U$ ranges over the $r$-tuples of the $G^{(n)}$ which contain $S$ as a subset; the ralency of an s-tuple is the number of $(r-s)$-tuples in its star. The product of an r-graph $A^{(1)}$ and an s-graph $B^{(s)}$ will be an $(r+s)$-graph whose vertex set is the union of those of $A^{(r)}$ and $B^{(s)}$ and whose $(r+s)$-tuples are all unions of an $r$-tuple of the first and a disjoint s-tuple of the second; in particular, a cone over $A^{(n)}$ is a product of $A^{(n)}$ with a disjoint $G^{(1)}(1 ; 1)$; a double pyramid (mentioned above) is a product of a polygon (graph) with a disjoint $G^{(1)}(2: 2)$.

It will be helpful to use geometrical language from time to time interpreting the triples of a 3-graph as the 2-simplexes of a simplieial 2-complex (which contains all possible 1-simplexes). A wheel will be a cone over a polygon. An octahedron will be a double pyramid over a 4-gon.

3. An elementary result on wheels. let $W$ denote the set of all wheels.

Thвокем 1. $\lim n^{-2} \operatorname{ex}(n ; W)=1 / 3$.

Proof. $n \rightarrow-$

a. In any $G^{(3)}(n ;[n(n-2)+1] / 3)$ some vertex must have valency at least $n-1$, hence its star must contain a polygon. Thus for all $n$, ex $(n ; W)<$ $<(n-1)^{2 / 3}$.

b. We construct for every integer $n$ a 3 -graph $A_{n}^{(3)}(n ; n(n-4) / 3)$ containing no wheelk. The vertices will be the residues modulo $n ;\{x, y, z\}$ is selected as a triple if and only if $x+y+z=0$ or $1(\bmod n)$, and $x, y, z$ are distinct. For fixed $x$, the number of selutions $(x, y, z)$ of these congruences ix $2 n$; of these, at most four are of the form $(x, y, y)$, and at most two of each of the forms $(x, y, x)$ and $(x, x, z)$. Thus the number of triples in the 3-graph is at least $n(2 n-8) / 3$ !. In the star of any vertex $x$, every vertex has valency at most 2. Any polygon there is of the form $y_{1}+y_{2}, \ldots, y_{f}, y_{1}$ where $y_{1}+y_{2}=11, x_{2}$ $y_{2}+y_{3} \equiv 1-x, y_{3}+y_{4}=-x$ ete. i.e. 
$\ldots=y_{3}-y_{3}=y_{3}-y_{1}=1=y_{2}-y_{4} \equiv y_{4}-y_{6} \equiv \ldots$. But this implies that all vertices, including $x$ are in the polygon, which is impossible. We conclude that the star is a union of open ares.

As a related result we have the following. Let $X^{(3)}$ denote the $G^{(3)}(4 ; 2)$ with two triples having an edge in common, i.e, the produet of a $G^{(2)}(2 ; 1)$ and $a$ disjoint $G^{(1)}(2 ; 2)$.

Тнвонем 2. $\lim _{n \rightarrow-} n^{-t} \operatorname{ex}\left(n: X^{(1)}\right)=1 / 6$.

Proor.

a. In any $G^{(3)}\left(n,\left(\begin{array}{l}n \\ 2\end{array}\right) / 3+1\right)$ some edge lies in more than one triple: this yields an upper bound of

$$
n^{-2} \operatorname{ex}\left(n ; X^{(3)}\right) \leq\left(1-n^{-1}\right) / 6 .
$$

b. The Steiner triple systems $[12,17]$ which exist for $n \equiv 1$ or $3(\bmod 6)$ $(n \geq 7)$, are example of a $G^{(3)}(n ; n(n-1) / 6)$ where every edge has unit valency. Lower bounds for the other congruence clases of $n$ are easily obtained from these 3 -graphs by ensing up to three vertices (and all triples containing them) from these 3-graphs.

4. Triangulations of the 2-sphere. Let $T$ denote the set of triangulations of the 2-sphere. (We rely on geometric intuition in part of the following discussion. A rigorous definition of a triangulation of the 2-sphere might be: a $G^{(x)}(n ; t)$ such that

(i) the star of every vertex is a polygon;

(ii) every edge is in zero or two triples;

(iii) (orientability) the triples can be each cyclically ordered so that the two orientations induced on any edge of valency two are in opposite directions:

(iv) (genus 0 ) the number of edges of valency 2 is exnetly $n+t-2$.)

Tнвовкм 3. There exid pasifive comstants $c_{1}$ and $c_{2}$ such that for all $n$.

$$
c_{1}<n^{-3,2} \operatorname{ex}(n ; T)<c_{2} \text {. }
$$

(In fact we prove that for $e>0$ and $n$ sufficiently large, $c_{1}>3^{-52}-\varepsilon$. $\left.c_{2}<18^{-12}+t\right)$.

Proof.

a. The upper bound: Let $c$ be a constant (to be further specified below). The sum of the valencies of the edges of $a G^{(a)}\left(n, \mathrm{~cm}^{52}\right)$ is at lenst $3 \mathrm{cn}^{5 z}$; hence the number of unordered pairs of triples having an edge in common must be at 
least $\frac{1}{2}\left(\left(3 c n^{5 / 2}\right)^{2} /\left(\begin{array}{l}n \\ 2\end{array}\right)-3 c n^{5 / 2}\right)$ whieh is greater than $(n-3)\left(\begin{array}{l}n \\ 2\end{array}\right)$ when $c>$ $>\left(n^{\frac{1}{2}}+\left(8 n^{2}-31 n+24\right)^{\frac{1}{4}}\right) / 12 n$, in particular when $c>18^{-\frac{1}{t}}+(144 n)^{-\frac{1}{4}}$, For such $c$ there must exist an edge $x y$ whose star contains at least $n-2$ edges. hence contains a polygon. The product of this polygon with the $G^{(1)}(2 ; 2)$ formed by $x$ and $y$ is a double pyramid.

b. The lower bound: Let $q$ be a prime power and $m=q^{2}+q+1$, We construct a 3-graph $S_{q}^{(3)}(3 m, m(q+1))$ as follows. Let $A$ denote the set of residues modulo $m$. By a well known theorem of SingizR [19] there exists a perfect differenee set $D$ for $A$, i.e. a subset $D$ of $A$ such that every non-zero element of $A$ is uniquely representable modulo $m$ as a difference $d_{1}-d_{2}$ where $d_{1}, d_{2} \in D$. The vertex set of $S_{q}^{(3)}$ will be the union of three disjoint copies of $A$, labelled $A_{1}, A_{2}, A_{3}$. The triples will be precisely those $\left(a_{1}, a_{2}, a_{3}\right)$ with $a_{i} \in A_{i}$ $(i=1,2,3)$ and $a_{1}+a_{2}+a_{3} \in D$. (As always, addition is modulo $m$.) Suppose now that $M^{(3)}$ is a sub-3-graph of $S_{q}^{(3)}$ whose every vertex has a polygon for its star. Without limiting generality, consider a vertex $a_{1}$ in $A_{1}$. Its star must be an even polygon of the form $a_{2}^{1}, a_{3}^{1}, a_{2}^{2}, a_{3}^{2}, \ldots, a_{2}^{r}, a_{2}^{r}, a_{2}^{1}$ where $a_{2}^{i} \in A_{2}, a_{3}^{i} \in A_{3}$. Suppose $r=2$, i.e. that $a_{1}$ has valency 4 . Then the following triples must have their sums in $D: a_{1} a_{2}^{1} a_{3}^{1}, a_{1} a_{3}^{1} a_{2}^{2}, a_{1} a_{2}^{2} a_{3}^{2}, a_{1} a_{3}^{2} a_{2}^{1}$. Distinctness of the vertices of the polygon forces the four sums here to be distinct. But then $a_{3}^{2}-a_{3}^{1}=$ $=\left(a_{1}+a_{2}^{i}+a_{3}^{2}\right)-\left(a_{1}+a_{2}^{i}+a_{3}^{1}\right)(i=1,2)$ a contradiction, in that a nonzero element cannot be expressible in two ways as a difference of elements of the difference set. Thus the only triangulations $M^{(3)}$ of surfaces contained in $S_{q}^{(3)}$ must have all vertices of valency at least 6. But it is well known ([13], p. 104) that any triangulation of the sphere must include vertices of valency less than 6. Thus ex $(3 m, T)>m^{5 / 2}$.

Let now $\varepsilon$ be given between 0 and 1. For $n$ sufficiently large there exists a prime $p$ between $\left((1-E)^{2 / 5} n / 3-3 / 4\right)^{1 / 3}-1 / 2$ and $(n / 3-3 / 4)^{1 / 2}-1 / 2$, i.e. $(1-\varepsilon)^{2 / 5} n<3\left(p^{2}+p+1\right)<n$. We may construct a 3 -graph with $n$ vertices by adjoining isolated vertices to $S^{(3)}$. The resulting $G^{(3)}(n)$ has more than $(1-\varepsilon) 3^{-5 / 2} n^{5 / 2}$ triples and contains no triangulation of the sphere.

5. Further extremal problems for 3-graphs. We now commence a study similar to that begun for graphs in [4]. Namely, if $\oint_{g}^{(r)}(l ; k)$ denote the set of all $r$-graphs $G^{(t)}(l ; k)$, we inquire as to value of $\operatorname{ex}\left(n, \mathcal{C}^{(3)}(l ; k)\right)$, which value we denote by $f^{(3)}(n ; l, k)-1$ to be consistent with [4]. Below we determine the asymptotio behavior of some of these numbers for $l \leq 6$.

Theorem 4. (The symbols $c_{3}, c_{4}, \ldots$ will denote positive consfants whase value we may estimate in the course of our proofs.)

a. $\lim _{n \rightarrow \infty} n^{-2} f^{(3)}(n ; 4,2)=1 / 6$

$$
c_{3} n^{3}<f^{(3)}(n ; 4,3)<f^{(3)}(n ; 4,4)
$$


b.

$$
\begin{aligned}
& f^{(3)}(n ; 5,2)=[n / 3]+1 \\
& c_{4} n^{2}<f^{(3)}(n ; 5,3)<c_{5} n^{2} \\
& c_{6} n^{5 / 2}<f^{(3)}(n ; 5,4)<c_{7} n^{5 / 2} \\
& c_{8} n^{3}<f^{(3)}(n ; 5,5)<\ldots<f^{(3)}(n ; 5,10) \\
& \text { c. } \quad f^{(3)}(n ; 6,2)=2 \\
& c_{9} n^{3 / 2}<f^{(3)}(n ; 6,3) \\
& c_{10} n^{2}<f^{(3)}(n ; 6,4)<(1 / 4) n^{2} \\
& f^{(3)}(n ; 6,6)<c_{11} n^{5 / 2} \\
& f^{(3)}(n ; 6,8)<c_{12} n^{11 / 4} \\
& c_{13} n^{3}<f^{(3)}(n ; 6,9)<\ldots<f^{(3)}(n ; 6,20) \text {. }
\end{aligned}
$$

Proof, a.The first statement is simply Theorem 2. To prove the second we need only consider the 3 -graph we call $T^{(3)}\left(n,\left[n^{3 / 27}\right]\right)$ defined as follows: the vertices are partitioned into classes $A, B, C$, containing respectively $[n / 3]$, $[(n+1) / 3]$, and $[(n+2) / 3]$ members; the triples are all $\{a, b, c\}$ such that $a \in A, b \in B, c \in C$. The case $l=k=4$ is the first open case of Turán's problem [20].

b. $k=2$. The lower bound follows from the $G^{(3)}(n,[n / 3])$ which has no two triples sharing a vertex. And, in any $G^{(3)}(n,[n / 3]+1)$, some vertex must have valency exceeding 1 .

$k=3$. The lower bound follows from Theorem 2; the upper from Theorem 1. However, some improvement in the constant of the upper bound is possible; cf, the techniques used in proving the case $l=6 \mathrm{k}=4$.

$k=4$. The lower bound follows from the 3 -graphs $S_{q}^{(3)}$ constructed in the proof of Theorem 3. Any $G^{(3)}(5 ; 4)$ contained in $S_{q}^{(3)}$ must have the property that its vertices ean be partitioned into three classes so that every triple contains one vertex from each class. It is easily seen by inspection that the only $G^{(3)}(5 ; 4)$ with this property is a cone over a quadrangle. But we saw in the proof of Theorem 3 that $S_{q}^{(3)}$ cannot contain such a wheel. The remainder of the proof of the lower bound, viz. the passage from special values of $n$ to all $n$ sufficiently large, is analogous to that used in the proof of Theorem 3 .

In any $G^{(3)}\left(n ; c n^{5.2}\right)$ some vertex $x$ must have valency more than $3 c(n-1)^{3 / 2}$. By a result of Kövíi-Sós-TuRáx [15] a sufficiently large constant $c$ ensures the existence in the star of $x$ of a 4-gon; hence the 3-graph contains a cone over a 4 -gon. (The other examples of a $G^{(3)}(5 ; 4)$ can all be shown to require (constant) $n^{3}$ triples - by the examples $T^{(3)}$ constructed in the proof of a. above.) 
$k=5$. The lower bound follows from the examples $T^{(3)}$ of a, above.

c. The case $k=2$ is trivial.

$k=3$. To prove the lower bound we construct a family of examples $G^{(3)}(n)$ for $n=q^{2}+q+1$ ( $q$ any odd pritne power) having for $q$ sufficiently large, more than $(1 / 6) n^{3}$ triples but containing noG(a) $(6 ; 3)$; the proof then may be completed as in the proof of the lower bound in Theorem 3. Our example is the 3-graph "spanned" by the graph exploited in [1], $\$ 3$ and [6], Theorem 1. The vertices will be the points of a finite projective plane $P G(2, q)$. Let $\lambda$ be an orthogonal polarity. A triple $\{x, y, z\}$ of points is selected if and only if the points are distinct and pairwise conjugate with respect to $\lambda$ i.e. each is incident with the polars of the other two, (sometimes ealled a self-polar triangle). It is casily seen that no triple selected can contain an absolute point of the polarity. (We could "improve" our example by discarding these points, but this improvement will be of no importance asymptotically.) No two distinct triples can have two vertices in common, for the polar line of any point of a triple is determined by the other two. Hence, if this 3-graph is to contain a $G^{(2)}(6 ; 3)$, that 3 -graph must be of the following form: $V=\left\{1,2,3,1^{\prime}, 2^{\prime}, 3^{\prime}\right\}$ $E=\left\{1^{\prime} 23,12^{\prime} 3,123^{\prime}\right\}$. The existence of such a sub-3-graph would imply that 1,2 and 2,3 and 3,1 are each conjugate pairs, i.e. that 123 is a triple. But that would yield several pairs of triples sharing an edge; which, as we have remarked above, cannot exist. Every line $l$ of the geometry which is not incident with its pole contains at least $q-1$ points not on the conic of the polarity. Any two of these points, together with the pole of $/$ form a selected triple. The number of non-4elf-conjugate lines is $q^{2}$ : the number of such triples is $\left(q^{2} / 3\right)\left(\begin{array}{c}q-2 \\ 2\end{array}\right)>$ $>(1 / 6)(1-\varepsilon) q^{4}$ for $q$ sufficiently large $(0<\varepsilon<1$ arbitrary $)$.

We have not kucceeded in establishing an upper bound better than (constant) $x^{*}$.

$k=4$. To establish a lower bound (probably capable of some improvement in the constant) let the vertices of a $G^{(3)}(n)$ be the residues modulo $n$. A triple $x y z$ is selected if and only if $x, y, z$ are distinct, and $x+y+z \equiv 0$ modulo $n$. This 3 -graph has at least $n-2$ admissible triples containing $x$, hence at least $n(n-2) / 6$ triples in all. No two triples share two vertices, since the third is uniquely determined by any two. Thus the only type of $G^{(1)}(6 ; 4)$ which could be present would be of the following form:

$$
\begin{aligned}
& V=\left\{1,2,3,1^{\prime}, 2^{\prime}, 3^{\prime}\right\} \\
& E=\left\{1^{\prime} 23,12^{\prime} 3,123^{\prime}, 1^{\prime} 2^{\prime} 3^{\prime}\right\} .
\end{aligned}
$$

However, the four congruences implied by the existence of these four triples imply the congruence for 123, which is impossible since no two triples share an edige! 
For an upper bound we apply (4) of [4]. In any $G^{(3)}(n ;(n[(3 / 4)(n-1)]+$ $+1)(3)$ some vertex has a $G(n-1 .[(3 / 4)(n-1)]+1)$ as a star; hence the star contains a $G(5 ; 4)$. It seems likely that the lower bound could be improved to allow the establishment of $\lim n^{-2} f^{(3)}(n ; 6,4)=1 / 4$.

$k=6$. Let a $G^{(x)}\left(n ; c_{11} n^{5}\right)$ be given, where the constant is to be further specified below. By the same sort of reasoning as used in the prof of the upper bound of Theorem 3, there are at least $9 c^{2} n^{3}\left(1-(6 c \mid \vec{n})^{-1}\right)$ (pairs of triples sharing an edge, which is more than $9 c^{2}(1-\xi) n^{3}$ when $n>\left(36 c^{3} \varepsilon^{2}\right)^{-1}$ ( $\varepsilon$ being any real number strictly between 0 and 1 ). Hence for such $n$ there exist two vertices whose stars have more than $18 c^{2}(1-\varepsilon) n$ edged in common; by ([4], (4)) these stars have a $G(4 ; 3)$ in commen if $18 c^{2}(1-\varepsilon) \geq 2 / 3$, with $n$ restricted, for example, to exceed $e^{-4}$.

$k=8$. Let a $G^{(3)}\left(n, c_{12} n^{114}\right)$ be given, where the constant is to be further specified below. As in the case $k=6$ above, there are at least $9 c^{2} n^{72}(1$ - $\left.(6 c \sqrt{n})^{-1}\right)$ pairs of triples sharing an edge. Hence for $0<\varepsilon<1$ and $n>\left(36 c^{2} \varepsilon^{2}\right)^{-1}$ there exist two vertices whose stars have more than $18 c^{2}(1-\varepsilon) n^{32}$ edges in common. The proof concludes as in the earlier case. using the result of Kövári-Sós-TuRix [15] cited earlier.

$k=9$. Any 3 -graph $G^{(3)}(6)$ with vertices in three classes such that any triple has one vertex in each class can have no more than eight triples. Thus the 3 -graphs $T^{(3)}$ constructed in the proof of $n$. yield a lower bound.

\section{REFERENCES}

[1] W. G. Brown, On graphs that do not contain a Thomsen graph. Carad. Math. Bull. $9(1966), 281-285$.

[2] P. Ereős and A. H. Srove, On the strueture of linear graphs, Buh. Amer. Math. Soc. 52 (1946), $1087-1091$.

[3] P. Eroós and T. Garrax, On maximal paths and eireuits of graphs, Acta Math. Acad. Sci. Hungar. 10 (1959), 337-356.

[4] P. Erpös, Extremal problems in graph theory, Theory of Graphs and its Applications (Proc. Sympos. Rmolenice, 1963), Prague, 1964, 29-36.

[5] P. Eroós, On extremal problems of graphs and generalized graphs, Israel $J$. Math. $2(1964), 183-190$.

[6] P. Erdós, A. Rx́nyr and V. T. Sós, On a problem of graph theory, Studia Sci. Math. Hungar. 1 (1966), 215235.

[7] P. EnDös and M. Snroxoyrrs, A limit theorem in graph theory, Studia Sei. Math. Hungar, 1 (1966), $51-57$.

[8] P. Eroós and M. Snmonovits, Some extremal problems in graph theory, Combinatorial Theory and its A pplications (Colloq. Math. J. Bolyai 4), Amaterdam-London, $1970,377-390$.

[9] P. ERDós and D. J. Kuerrutax, On eoloring graphs to maximize the proportion of multicolored k-edges, J. Combinatorial Theory 5 (1968), 164-169.

[10] P. Erdós and V. T. Sós, Some remarks on Ramsey's and Turán's theorem, Oombinatorial Theory and its Applications (Colloq. Math. J. Bolyai 4), AmsterdimLondon, 1970, $395-401$.

[11] P. ERDōs, On some extremal properties on r-graphs, Discreto Math, 1 (1971), 1 -6.

[12] M. K. Forx, JR, and G. A. Henuesd, Minimal eoverings of pairs by triples, Pacifie J. Math. 8 (1958), $709-719$.

[13] F. Harsax, Graph Theory, Reading, Mass, 1969. 
[14] Gy. Katona, T. Nemetz and M. Smonovirs, Ujabb bizonyitís a Turán-féle gráftételre és megjegyzések bizonyos általánositásaira, Mat. Lapok 15 (1964), 228 - 238.

[15] T. Kövári, V. T. Sós and P. Tur Á, On a problem of K. Zarankiewiez, Colloq. Math. $3(1955), 50-57$.

[16] G. Rincast, Extremal problems in the theory of graphs, Theory of Grapha and its Applications (Proc. Sympos. Smolenice, 1963). Prague, 1964, 85-90.

[17] H. Rxsier, Combinatorial Mathematics, New York, 1963.

[18] M. Srsovovms, Extremal graph problems with conditions, Combinatorial Theory and its Appl. (Colloq. Math. J. Bolyai 4), Amsterdam-London, 1970, 999-1011.

[19] J. SrveEn, A theorem in finite projective geometry and some applieations to number theory, Trans. Amer. Math. Soc. 43 (1938), 377-385.

[20] P. Tunáv, Egy gráfelméleti szélsórté̉k-feledatról, Mat. Fí. Lapok 43 (1941), $436-452$.

[21] P. Torán, On the theory of graphs, Collog. Math. 3 (1954), $19-30$.

\section{(Received December 20, 1971)}

BOTYOS LOHAND TUDOMENYTGGYUTHM

ANATIZIS I. TANBZRK

$H-108 B$ BUDAPBST

MUZEUM KBT, $6-8$.

HUNGARY

MTA MATRMATYKAI KUTATO INTFEETE

H-1058 BUDAPEST

IEKTTANODA U, 13-15.

DEPATTMENT OR MATHEMATICS

MeGII. UNIVBRSTTY

DIONTREAT, P, Q

CANADA 